\title{
A NOTE ON MATRIX SUMMABILITY OF A CLASS OF FOURIER SERIES
}

\author{
BY BADRI N. SAHNEY 1
}

Communicated by Murray H. Protter, June 2, 1969.

1. Recently several papers by Rajagopal [7] ,Varshney [11] and others have been written, on Nörlund summability of Fourier series, in order to unify some of the classical results. Though lower semitriangular matrix $(\Lambda)$ summability method has been known for quite some time no attempt has yet been made to apply it to Fourier series. The object here is to determine a necessary and sufficient condition for $(\Lambda)$ summability of Fourier series and to include a wider class of known results.

A Fourier series, of a Lebesgue-integrable function, is said to be summable at a point by triangular matrix method $(\Lambda)$, defined by Hardy [1], if $\Lambda_{n, k}=0$ for $k>n, \sum \Lambda_{n, k} \rightarrow 1$ as $n \rightarrow \infty$ and $\sum_{k=0}^{n}\left|\Lambda_{n, k}\right| \leqq M$, where $M$ is a constant, and the point is in a certain subset of the Lebesgue set.

The following main theorem has been proved here.

THEOREM. Let a sequence $\left\{\Lambda_{n, k}\right\}$ be defined in terms of

$$
\begin{aligned}
& \Lambda_{n}(u), \text { monotonic decreasing and strictly positive for all } u \geqq 0, \\
& \Lambda_{n, u} \equiv \Lambda_{n}(u)
\end{aligned}
$$

and if

$$
\Phi(t) \equiv \int_{0}^{t}|\phi(u)| d u=o\left(\frac{t}{\psi(1 / t)}\right) \text { as } t \rightarrow+0
$$

and $\psi(t)$ be positive, nondecreasing with $t$; then a necessary and sufficient condition for $(\Lambda)$ summability of Fourier series, to 0 or

$$
t_{n} \equiv\left\{\sum_{k=0}^{n} \Lambda_{n, b} S_{k}\right\} \rightarrow 0 \text { as } n \rightarrow \infty,
$$

is

$$
\int_{1}^{n} \frac{\bar{\Lambda}_{n}(u)}{u \psi(u)} d u=O(1)
$$

1 The author wishes to thank Canadian Mathematical Congress for the support of this work, through Summer Research Institute at Winnipeg. The author also wishes to thank Professor L. S. Bosanquet for many valuable suggestions, during the author's brief stay at University College, London. 
where $\bar{\Lambda}_{n, k} \equiv \sum_{r=1}^{k} \Lambda_{n, r} \equiv \bar{\Lambda}_{n, k}(k)$.

2. We shall need the following lemma.

LEMma. If the sequence $\left\{\Lambda_{n, k}\right\}$ is defined as in (1.1), then

$$
\left|\frac{\sum_{k=0}^{n} \Lambda_{n, k} \operatorname{Sin}\left(k+\frac{1}{2}\right) u}{\operatorname{Sin}(u / 2)}\right|<c \frac{\bar{\Lambda}_{n}(1 / u)}{u},
$$

where $c$ is a constant, not the same at each occurrence.

Proof. If we choose $m=$ integral part of $1 / u$ and suppose that $1 / n \leqq u \leqq \delta$, we get $m \operatorname{Sin} \frac{1}{2} u>m u / \pi$. Now for $u>0$ and $m \leqq n$ we have

$$
\begin{aligned}
& \left|\frac{\sum_{k=0}^{n} \Lambda_{n, k} \operatorname{Sin}\left(k+\frac{1}{2}\right) u}{\operatorname{Sin}(u / 2)}\right| \\
& \quad<\frac{1}{\operatorname{Sin} \frac{u}{2}}\left[\left|\sum_{k=0}^{m} \Lambda_{n, k} \operatorname{Sin}\left(k+\frac{1}{2}\right) u\right|+\left|\sum_{m}^{n} \Lambda_{n, k} \operatorname{Sin}\left(k+\frac{1}{2}\right) u\right|\right] \\
& \quad<\frac{1}{\operatorname{Sin} \frac{u}{2}}\left[\sum_{k=0}^{m} \Lambda_{n, k}\left|\operatorname{Sin}\left(k+\frac{1}{2}\right) u\right|+\bar{\Lambda}_{n, m} \operatorname{Max}_{m \leqq k \leqq n} \sum_{m}^{n} \operatorname{Sin}\left(k+\frac{1}{2}\right) u\right] \\
& =\frac{\bar{\Lambda}_{n, m}}{\operatorname{Sin} \frac{u}{2}}+\Lambda_{n, m}{ }_{m \leqq k \leqq n}^{\operatorname{Max}} \frac{\operatorname{Cos}\left(k+\frac{1}{2}\right) u}{\operatorname{Sin} \frac{u}{2}} \\
& <\frac{\bar{\Lambda}_{n}(m)}{\operatorname{Sin} \frac{1}{2} u}+\frac{c \bar{\Lambda}_{n}(m)}{m\left(\operatorname{Sin} \frac{1}{2} u\right)^{2}} \\
& =\frac{\bar{\Lambda}_{n}[1 / u]}{\operatorname{Sin} \frac{1}{2} u}+\frac{c \bar{\Lambda}_{n}[1 / u]}{m\left(\operatorname{Sin} \frac{1}{2} u\right)^{2}} \\
& <\frac{\bar{\Lambda}_{n}[1 / u]}{\operatorname{Sin} \frac{1}{2} u}+\frac{c \bar{\Lambda}_{n}[1 / u]}{\operatorname{Sin}\left(\frac{1}{2} u\right)} \\
& <\frac{c \bar{\Lambda}_{n}(1 / u)}{u},
\end{aligned}
$$

which proves the lemma.

3. Proof of the theorem. To prove the sufficiency part, first, we see [2] that the $n$th partial sum of Fourier series is given by 


$$
\begin{aligned}
S_{n} & =\frac{1}{\pi} \int_{0}^{\pi} \phi(u) \frac{\operatorname{Sin}\left(n+\frac{1}{2}\right) u}{\operatorname{Sin} \frac{1}{2} u} d u \\
& =\frac{1}{\pi} \int_{0}^{\delta} \phi(u) \frac{\operatorname{Sin}\left(n+\frac{1}{2}\right) u}{\operatorname{Sin} \frac{1}{2} u} d u+o(1) .
\end{aligned}
$$

Using (1.3), and the last expression, we get

$$
\begin{aligned}
t_{n} & =\frac{1}{\pi} \int_{0}^{\delta} \phi(u) \sum_{k=0}^{n} \Lambda_{n, k} \frac{\operatorname{Sin}\left(k+\frac{1}{2}\right) u}{\operatorname{Sin} \frac{1}{2} u} d u+o(1) \\
& =\frac{1}{\pi}\left[\int_{0}^{1 / n}+\int_{1 / n}^{\delta}\right] \phi(u) \sum_{k=0}^{n} \Lambda_{n, k} \frac{\operatorname{Sin}\left(k+\frac{1}{2}\right) u}{\operatorname{Sin} \frac{1}{2} u} d u+o(1) \\
(3.1) \quad & =I_{1}+I_{2}+o(1), \text { say, }
\end{aligned}
$$

\section{by virtue of (1.1).}

Considering $I_{1}$, we get

$$
\begin{aligned}
I_{1} & =\frac{1}{\pi} \int_{0}^{1 / n} \frac{\phi(u)}{\operatorname{Sin} \frac{1}{2} u} \sum_{k=0}^{n} \Lambda_{n, k} \operatorname{Sin}\left(k+\frac{1}{2}\right) u d u \\
& =O(n) \int_{0}^{1 / n}|\phi(u)| d u \\
& =o\left(\frac{1}{\Psi(n)}\right), \quad \text { by (1.2). }
\end{aligned}
$$

Next, by the above lemma

$$
\begin{aligned}
I_{2}= & O(1) \int_{1 / n}^{\delta}|\phi(u)| \frac{\sum_{k=0}^{n} \Lambda_{n, k} \operatorname{Sin}\left(k+\frac{1}{2}\right) u}{\operatorname{Sin} \frac{1}{2} u} \mid d u \\
= & O(1) \int_{1 / n}^{\delta}|\phi(u)| \frac{\bar{\Lambda}_{n}(1 / u)}{u} d u \\
= & O(1)\left\{\left[\Phi(u) \frac{\bar{\Lambda}_{n}(1 / u)}{u}\right]_{1 / n}^{\delta}+\int_{1 / n}^{\delta} \Phi(u) d\left[\frac{\bar{\Lambda}_{n}(1 / u)}{u \Psi(1 / u)} \cdot \Psi\left(\frac{1}{u}\right)\right]\right\} \\
= & o\left(\frac{\bar{\Lambda}_{n}(n)}{\Psi(n)}\right)+o(1) \\
& +o(1) \int_{1 / n}^{\delta} \frac{\bar{\Lambda}_{n}(1 / u)}{\{\Psi(1 / u)\}^{2}} d \Psi\left(\frac{1}{u}\right)+o(1) \int_{1 / n}^{\delta} u d\left\{\frac{\bar{\Lambda}_{n}(1 / u)}{u \Psi(1 / u)}\right\} \\
= & o(1)+o(1) \int_{1 / n}^{\delta} \frac{d \Psi(1 / u)}{\{\Psi(1 / u)\}^{2}}+o(1) \int_{1 / n}^{\delta} \frac{\bar{\Lambda}_{n}(1 / u)}{u \Psi(1 / u)} d u \\
= & o(1), \quad \text { by virtue of }(1.4) .
\end{aligned}
$$


Now the first part of the proof is complete by virtue of (3.1), (3.2) and (3.3), when $n \rightarrow \infty$.

To prove the necessary part, a look at the proof of sufficiency part shows that, it is sufficient to show here that

$$
\int_{1 / n}^{\delta} \frac{u}{\Psi(1 / n)} d\left\{\frac{\bar{\Lambda}_{n}(1 / u)}{u}\right\}=O(1) .
$$

Considering the left-hand side and proceeding as above, we have

$$
\begin{aligned}
\int_{1 / n}^{\delta} \frac{u}{\Psi(1 / u)} & \left.d \frac{\bar{\Lambda}_{n}(1 / u)}{u}\right\} \\
& =O(1) \int_{1 / n}^{\delta} u d\left\{\frac{\bar{\Lambda}(1 / u)}{u \Psi(1 / u)}\right\}+O(1) \int_{1 / n}^{\delta} \frac{\bar{\Lambda}_{n}(1 / u)}{\{\Psi(1 / u)\}^{2}} d \Psi(1 / u) \\
& =O\left(\frac{1}{\Psi(n)}\right)+O\left(\bar{\Lambda}_{n}(n)\right)+O(1) \int_{1 / n}^{\delta} \frac{\bar{\Lambda}_{n}(1 / u)}{u \Psi(1 / u)} d u
\end{aligned}
$$

which is bounded by virtue of (1.4) and this completes the proof.

4. The results due to Hille and Tamarkin [4], Iyengar [6] and Siddiqi [10] on harmonic summability form the particular cases of the theorem for $\Lambda_{n, k} \equiv(1 / \log (n+1)) \cdot(1 /(k+1))$ and $\Psi(1 / t) \equiv \log (1 / t)$. The case on $(H, p)$-summability due to Sahney [8] can be obtained by considering the case

$$
\Psi(u)=\prod_{q=0}^{p-1}(\log )^{q+1}(u)
$$

and

$$
\Lambda_{n, k}=\frac{1}{(\log )^{p}(n+1) \prod_{q=0}^{p-1}(\log )^{q}(k+1)} .
$$

Results on Cesàro summability, due to Fejer, Lebesgue and Hardy [2] can be obtained if we choose $\Psi(u) \equiv 1$ and

$$
p_{n}=\frac{\sqrt{n+\alpha}}{\sqrt{(n+1)} \sqrt{\alpha}}
$$

for $0<\alpha<1$ and $p_{n}$ is as defined below.

Lastly the different results on Nörlund summability, for $\Lambda_{n, k}$ $=p_{n-k} / P_{n}$, can be obtained by considering the cases $\Psi(u) \equiv 1$ and $\Psi(u) \equiv \log u$ which are due to Hille-Tamarkin [4], Rajagopal [7] and Varshney [11], respectively. 


\section{REFERENCES}

1. G. H. Hardy, Divergent series, Oxford, 1949.

2. G. H. Hardy and J. E. Littlewood, Notes on the theory of series. XVIII. On the convergence of Fourier series, Proc. Cambridge Philos. Soc. 31 (1935), 317-323.

3. E. Hille and J. D. Tamarkin, On the summability of Fourier series, Proc. Nat. Acad. Sci. 14 (1928), 915-918.

4. - On the summability of Fourier series, Trans. Amer. Math. Soc. 34 (1932), 757-783.

5. K. S. K. Iyengar, New convergence and summability tests for Fourier series, Prod. Ind. Acad. Sci. Sec. A 18 (1943), 113-120.

6. - Notes on the summability. II. On the relation between summability by Nörlund means of a certain type and summability by Valiron means, Half Yearly J. Mysore Univ. Sec. B 4 (1944), 161-166.

7. C. T. Rajagopal, Nörlund summability of Fourier series, Proc. Cambridge Philos. Soc. 59 (1963), 47-53.

8. B. N. Sahney, On the $(\mathrm{H}, p)$ summability of Fourier series, Boll. Un. Mate. Ital. 16 (1961), 156-163.

9. - On the Nörlund summability of Fourier series, Pacific J. Math. 13 (1963), 251-262.

10. J. A. Siddiqi, On the harmonic summability of Fourier series, Proc. Ind. Acad. Sci. Sec. A. 28 (1949), 527-531.

11. O. P. Varshney, On the Nörlund summability of Fourier series, Acad. Roy. Belg. Bull. Cl. Sci. (5) 52 (1966), 1552-1558.

12. A. Zygmund, Trigonometric series, 2nd ed., Chelsea, New York, 1952.

The University of Calgary, Alberta, Canada 